Disclosure of Interest None declared.

\section{P051/001 CHARACTERIZATION OF THE ANTI-CENTROMERE ANTIBODY RESPONSE IN SYSTEMIC SCLEROSIS PATIENTS SUGGESTS A BROAD AND ACTIVE B CELL RESPONSE}

${ }^{1} \mathrm{CM}$ Wortel ${ }^{*},{ }^{1} \mathrm{NM}$ van Leeuwen, ${ }^{1} \mathrm{M}$ Boonstra, ${ }^{2} \mathrm{JA}$ Bakker, ${ }^{1} \mathrm{RE}$ Toes, ${ }^{1} \mathrm{TW}$ Huizinga, ${ }^{1} \mathrm{JK}$ de Vries-Bouwstra, ${ }^{1} \mathrm{HU}$ Scherer. ${ }^{1}$ Rheumatology; ${ }^{2}$ Clinical Chemistry and Laboratory Medicine, Leiden University Medical Center, Leiden, Netherlands

\subsection{6/annrheumdis-2018-EWRR2019.43}

Career situation of first and presenting author Student for a master or a $\mathrm{PhD}$.

Introduction Systemic Sclerosis (SSc) is a rare, heterogeneous autoimmune disease characterized by microvascular damage, organ fibrosis and immune dysfunction. Autoantibodies are detected in $>95 \%$ of patients, the most prevalent being anticentromere (ACA) and anti-topoisomerase (ATA) antibodies. Although used for diagnosis, little is known about the underlying auto-reactive B cell responses. In particular, the ACA B cell response has been poorly studied.

Objectives Characterization of the ACA B cell response in SSc patients.

Methods ACA IgG, IgA and IgM levels were measured in serum samples of 167 ACA IgG + SSc patients. Patients were divided in a SSc (fulfilling ACR 2013 criteria, $n=132$ ) and a very early SSc group (fulfilling VEDOSS criteria, $n=35$ ). Additionally, PBMCs from ACA IgG+ SSc patients (and ATA IgG + SSc and healthy donors (HD) as control) were cultured either in the presence of CD40L expressing fibroblasts, IL-21 and BAFF or without stimulation. Levels of ACA IgG, IgA and IgM (and total Ig) were measured after one week of culture using ELISA.

Results ACA IgG+ SSc patients displayed a broad isotype usage with $75 \%$ being ACA IgA+ and $68 \%$ being ACA IgM + in serum. Patients within the SSc group showed higher ACA IgG levels and a higher percentage of ACA IgM positivity compared to the very early SSC group. ACA IgG, IgA and IgM could be measured in ACA SSc PBMC culture medium following stimulation, but not in ATA SSc and HD, indicating the presence of circulating ACA B cells of all three isotypes. In cultures that yielded sufficient Ig production, ACA IgG was detectable in 7/9 ACA SSc patients, ACA IgA in $3 / 7$ and ACA IgM in $2 / 7$. Furthermore, ACA IgG production was also detected in the absence of stimulation in 5/9 patients, suggesting the presence of ACA-producing plasmablasts in the circulation. No spontaneous production of antiTetanus Toxoid antibodies, a control recall response, was observed.

Conclusions ACA + SSc patients display a broad range of isotype usage in their ACA response, reflected both by ACA serum levels and presence of ACA IgG-, IgA- and ACA IgM-producing B cells in the peripheral blood. Additionally, ACA IgG production by unstimulated PBMCs points towards continuous differentiation of memory cells into antibody secreting cells. These data, together with differential isotype profiles between very early SSc and SSc patients, provide insight into the ACA B cell response and its potential involvement in disease-relevant pathogenetic processes.

Disclosure of Interest None declared.

\section{P052 AUTO-ANTIBODIES AGAINST POST TRANSLATIONAL MODIFIED PROTEINS IN OSTEOARTHRITIS: PILOT DATA COMPARING SYNOVIAL FLUID AND SERA}

${ }^{1} \mathrm{X} X \mathrm{Xie}{ }^{*},{ }^{2} \mathrm{MV}$ Delft, ${ }^{2} \mathrm{~L}$ Trouw, ${ }^{1} \mathrm{P}$ Conaghan, ${ }^{1} \mathrm{~F}$ Ponchel. ' ${ }^{2}$ Leeds Institute of Rheumatic and Musculoskeletal Medicine, The University of Leeds, Leeds, UK; ${ }^{2}$ Leiden University Medical Centre, Leiden University, Leiden, Netherlands

\subsection{6/annrheumdis-2018-EWRR2019.44}

Career situation of first and presenting author Student for a master or a $\mathrm{PhD}$.

Introduction Osteoarthritis (OA) is a highly prevalent disease and a leading cause of disability worldwide. OA is age-related and as such has a potential devastating impact on our ageing populations. The pathogenesis of $\mathrm{OA}$ remains poorly understood. Inflammatory responses are prevalent in driving processes associated with aging, and increased levels of IgG autoantibodies (auto-Abs) have been associated with age. In $\mathrm{OA}$, auto-Abs are also frequently observed, notably towards post translational modified (PTM) proteins. Such PTM are often the result of inflammation-driven mechanisms such as oxidative stress, carbamylation and citrullination ${ }^{1}$ in $\mathrm{OA}$ joints. Objectives To establish a profile of auto-Abs associated with PTM in OA and controls.

Methods Serum was collected from healthy controls (HC) and OA patients as well as rheumatoid arthritis (RA); knee synovial fluid (SF) samples were collected from OA/RA patients. All participants gave informed consent. The levels of antibodies against citrullinated protein antigens (ACPA) was measured (Phadia CCP-test), antibodies against carbamylated proteins (anti-CarP) were measure in collaboration with Leiden using an in-house ELISA, ${ }^{2}$ antibodies against glycated-collagen proteins (anti-ROS-CII) were analysed using an in house ELISA. ${ }^{3}$

Results ACPA positivity was present in 6\% $(n=24 / 392)$ of OA patients' serum, which was 3 times more frequent that in $\mathrm{HC}$ $(\sim 2 \%)$, but was present in $48 \%(n=22 / 46)$ of OA SF. RA frequencies were $73 \%(n=41 / 56)$ in SF and 65\% $(n=55 / 85)$ in serum. Anti-CarP auto-antibodies were detected in $11 \%(n=12 /$ $111)$ of OA sera and in $37 \%(n=127 / 340)$ of RA sera (SF results are not yet available). Anti-collagen auto-Abs in serum were observed at similar frequencies: $18 \%$ of OA $(n=6 / 34)$ and $18 \%(n=14 / 78)$ of RA patients, but only $6.7 \%(n=3 / 45)$ of OA fluids and $3.6 \%(n=2 / 56)$ of RA fluids were positive. Collagen modified by reactive oxygen species enables detection of auto-Ab (anti-ROS-CII); these were observed in 35\% $(n=12 / 34)$ of OA and $32 \%(n=25 / 78)$ of RA sera; this differed from SF with $20 \%$ positive $(n=9 / 45)$ in OA and $60 \%(n=34 / 56)$ in RA. Conclusions In $\mathrm{OA}$ patients, autoimmune responses against native proteins (especially collagens and proteoglycans) have been reported since the 1980 's, notably in relation with severity. ${ }^{4}$ However, our data demonstrate that auto-Abs may also result from the accumulation of PTMs induced by chemical reactions in the inflamed joint. These auto-Abs against PTM-antigens may also be contributing to disease severity, as these are notably frequently detected in SF even if a bit less frequently than in RA in our pilot cohorts.

\section{REFERENCES}

1. Burska AN. MI 2014;492873-492873.

2. Shi J, et al. ART 2015:17:339

3. Strollo $R$, et al. A\&R 2013;65:1702-12.

4. Yuan GH, et al. A\&R 2003;48:602-611.

Disclosure of Interest None declared 